\title{
Improving recovery time following heart transplantation: the role of the multidisciplinary health care team
}

This article was published in the following Dove Press journal:

Journal of Multidisciplinary Healthcare

20 August 2013

Number of times this article has been viewed

\author{
Maureen G Roussel' \\ Noreen Gorham ${ }^{2}$ \\ Lynn Wilson ${ }^{2}$ \\ Abeel A Mangi ${ }^{2}$
}

'Heart and Vascular Center, Yale-New Haven Hospital, New Haven, CT, USA; ${ }^{2}$ Center for Advanced Heart Failure, Mechanical Circulatory Support and Cardiac Transplantation, Yale New Haven Heart and Vascular Institute, Yale-New Haven Hospital, New Haven, CT, USA
Correspondence: Abeel A Mangi Section of Cardiothoracic Surgery, Yale University School of Medicine, 330 Cedar Street, Boardman Building 204, New Haven, CT 065 I0, USA

$\mathrm{Tel}+\mathrm{I} 2037855252$

Email abeel.mangi@yale.edu
Background: The care of cardiac transplant patients is complex requiring a finely orchestrated endeavor to save a patient's life. Given the chronic and complex nature of these patients, multiple disciplines are involved in their care. Recognizing difficulties with communication among team members and striving for improved efficiencies in our pretransplant listing process and in our inpatient care, our team was prompted to change the existing approach to patient care related to heart transplantation.

Methods: Daily multidisciplinary rounds were instituted and the format of the weekly Multidisciplinary Review Committee (MDRC) meetings was modified with the list of attendees broadened to include a larger interdisciplinary team. Additionally, the approach to patient care was analyzed for process improvement.

Results: The quality improvements are improved communication and throughput, quantified in an $85 \%$ decrease in time to complete transplant evaluation, a $37 \%$ decrease in median length of stay posttransplantation, and a $33 \%$ reduction in the 30 day readmission rate. In addition, pre- and posttransplant caregivers now participate in MDRC in person or via an electronic meeting platform to support the continuum of care. Quality metrics were chosen and tracked via a transparent electronic platform allowing all involved to assess progress toward agreed upon goals. These were achieved in an 18 month time period following the recruitment of new leadership and invested team members working together as a multidisciplinary team to improve the quality of cardiac transplant care.

Discussion: Implementation of daily multidisciplinary rounds and expansion of the attendees for the MDRC meetings improved care related to heart transplantation.

Keywords: multidisciplinary rounds, transplant, communication

\section{Introduction}

A multidisciplinary team approach to the care of the heart transplant patient has improved communication about care, empowered all members of the team to share observations and perspectives, and has enhanced the care team's ability to address patient issues - both salient and subtle - in real time. At the Yale-New Haven Hospital (YNHH), a 1,008 bed academic medical center in New Haven, CT, the positive effects of adhering to such a charter have become increasingly apparent.

Our center has incorporated the multidisciplinary approach to patient care with regular multidisciplinary rounds (MDR) as well as our listing process with a Multidisciplinary Review Committee (MDRC) with positive results. These include decreased time to transplant evaluation completion, decreased length of stay, and decreased 30 day readmissions, all of which support a fiscally responsible program. Both formats bring 
together various members of the team to coordinate patient care and to share expertise. ${ }^{1}$

Working as a multidisciplinary team has the potential to correct fundamental problems with patient care in the United States. The National Quality Forum reports that, indeed, the system is fragmented, complex and filled with inefficiencies, and that the failure to coordinate care can increase costs, errors, and complications. ${ }^{2}$ Further, when the care needed is both chronic and complex, the risks to quality and safety rise exponentially. The Institute for Healthcare Improvement's road map for quality and safety lists MDR, communication, and teamwork as essential to supporting excellent patient care. ${ }^{3}$

Until 2008, our institution, like many others, had followed the time honored tradition of daily physician led rounds. Each morning, the medical or surgical team "rounded" at the patient's bedside to discuss the patient's progress, test results, and plan of care for that day. Other team members often were not present ${ }^{4}$ and limited communication had the potential to result in errors. Later in the day, staff members in other disciplines would attend to the patient and leave notes in the medical record. Since heart transplant patients are complex and chronically ill, it is not unusual for them to have multiple "teams" following their care. If the surgeon who performed the heart transplantation was in the operating room at that time, it would be challenging if not impossible for teams to communicate with him/her.

We recognized that while there was plenty of constructive input into the care of the patient, there was minimal coordination of the input being delivered. Conflict resolution (when disagreements arose about the treatment regime) was difficult to accomplish. While daily morning rounds continue as a part of regular in-patient care at our hospital, the bedside MDRs have tremendously improved communication and care of heart transplant patients.

Transplantation is a field that by its very nature is unpredictable. Having a strong multidisciplinary team with consistent and reliable processes is crucial so that when an organ match becomes a reality the entire team is focused on a successful outcome both for the recipient and with respect to the donor and his/her family. Not only has our MDR process proven a benefit to patients, their families, and the multidisciplinary team, the literature supports the importance of teamwork and communication.

Coordinating care is paramount to decreasing errors, length of stay, and improving the patient experience. The systematic review of the literature "Organizational interventions to implement improvements in patient care: a structured review of reviews" by Wensing et $\mathrm{al}^{5}$ reviewed papers on palliative care and heart failure multidisciplinary teams and reported overall improvement in outcomes when compared with conventional care. Kim et al reported in 2010 the positive effect of significantly reduced risk of death associated with multidisciplinary care teams in intensive care units. ${ }^{6}$ Sen et al reported that daily multidisciplinary discharge rounds in a trauma center as time well spent as care was progressed in a timely manner. ${ }^{7}$ Although the authors focused on discharge planning, the paper demonstrates the importance of coordinated multidisciplinary team efforts.

Value based purchasing focuses on rewarding hospitals that meet (or penalizing those that fail to meet) specific metrics such as infection rates or hospital readmission rates. Hospitals across the nation are working to improve their care of patients under value based purchasing and in many cases are finding that coordination of the team providing care is the answer to improving their outcomes. These teams often have national, payer, or personal metrics to meet and using MDR is a powerful way to ensure progress toward desired outcomes. Our coordination of care has provided promising results, particularly in the last 18 months with our concerted focus on quality and safety.

The recent focus on safe handoffs in both the lay media and professional journal underscores the need for reliable team communication. The Agency for Healthcare Research and Quality (AHRQ) recognizes the safety hazards involved in discontinuity of care otherwise known as handoffs. Much is written about the need for accurate information exchange between caregivers to ensure patient safety. The Institute Of Medicine "Crossing the Quality Chasm" report identifies handoffs as cumbersome, and at times disorganized, with safety concerns related to loss of information that can hurt the patient. ${ }^{8}$ This is especially important with the increasingly complex, high acuity patients found in acute care hospitals today. In the field of cardiac transplant, the risks are even greater that missed communication could lead to inaccurate immunosuppression or other postoperative hemodynamic management issues.

For caregivers who can rely on regular MDRs there is the opportunity to clarify any concerns raised in handoff as the "entire team" is present and immediately available. If an individual caregiver has a question or does not know the patient well then another member of the team is sure to. This is not a benign concern. With multiple handoffs occurring each day and "new" clinicians joining the team of caregivers each shift, the need for someone to "know" the patient is paramount. A study by Gray and colleagues in a newborn intensive care unit 
found that by two weeks, $50 \%$ of the shifts were newcomer nurses who had not previously cared for the patient. ${ }^{9}$ They also described the difficulty in maintaining an accurate flow of information and seamless care during longer hospital stays that may include up to 300 nursing transitions/handoffs at shift changes. This study found parental satisfaction was increased when they had care provided by familiar faces more often, a finding that is not surprising. It is not unusual to hear clinicians caring for the transplant patients at our hospital saying, "I will address this at $2 \mathrm{pm}$ MDR rounds" for issues that do not require urgent resolution. Families also schedule visits around this time knowing they can find the whole team.

\section{Evolution of the "weekly transplant meeting" into the weekly "MDR"}

While the heart transplantation program at YNHH has been long-standing, with the first heart transplant performed in 1984, the multidisciplinary team had for many years been limited to a small core group of physicians, nurse coordinators, a hospital business office representative, and a dedicated transplant social worker. In the 1990s and through to early 2000 , these team members met weekly to review and discuss potential candidates for transplantation as well as to address any concerns surrounding the management of patients previously transplanted. Typically, either the cardiothoracic surgeon or the cardiologist would present the background on any candidate brought forth in consideration for heart transplantation. Oftentimes, formal evaluation of the candidate had been previously initiated by a physician's request to the nurse coordinator to "list" a patient for transplant. The primary role of the transplant nurse coordinator at the time of the meeting was to verify that all evaluation and laboratory testing as well as diagnostic studies had been completed. The social worker would ascertain presence of a support structure for the candidate, describe family dynamics pertinent to the prospect of cardiac transplantation, and identify barriers to moving forward. The business representative would address any financial constraints or limitations that would impact the feasibility of committing a patient and the Institution to transplantation. Although the ancillary team members described did have input in the discussion, unless major social or financial red flags were identified, the meeting would adjourn with a plan of action derived primarily from the recommendations of one or two key physicians.

Of note during this early period was the fairly independent approach utilized by the team physicians and ancillary consult services (such as social work, nutrition, physical therapists, and others) in managing heart transplant patients in the early postoperative period. Each discipline would round separately on the recipient, make an assessment based on that moment, and formulate an independent plan of care, the details of which would then be outlined in the patient's medical record. Invariably, this approach would lead to some fragmentation of care, as consensus agreement to treatment modalities or interventions often did not occur until there had been direct communication with the responsible attending physician. As a result, a certain level of frustration seemed to become a recurring theme among the health care team members charged with caring for many of the heart transplant recipients.

Those frustrations led to conversations on how best to improve dialogue around the plan of care. An initial solution was to include other cardiac transplant stakeholders with an invitation to the weekly MDRC meeting. These additional staff consisted of physical and occupational therapists, unit and floor based nurses, medical and surgical fellows, nurse practitioners, care coordinators, and on occasion, hospital administrators. Although not a stated goal, the primary impetus for this expansion seemed to be the need for improved communication about the decision making around this unique patient population.

The format of the weekly meetings went through several additional iterations in subsequent years, but did not completely evolve to its present state until change in both the program's medical and surgical directors occurred. The membership was further broadened to include additional important services previously only consulted on an ad hoc basis. These services included psychiatry, palliative care, nursing unit leadership, and staff nurses invested in the care of the patients being presented. These disciplines are now expected members at the meeting and attempt to participate as much as possible. A scripted clinical presentation format was introduced with the expectation that the transplant nurse coordinators present the patient to the entire multidisciplinary team. Removing the responsibility for patient presentation from the physicians was to allow for a most unbiased clinical portrait. A comprehensive clinical picture with all aspects of an individual's medical, social, and financial suitability is addressed in a standardized manner. Pre-meeting distribution of the anticipated agenda electronically allows all team members to be prepared to offer insight and observations pertinent to their expertise. Minutes of the meeting are distributed to all members ensuring accurate documentation of concerns, questions, and actions. These minutes include discussion points, any needed follow-up, and a summary of patients progressing through the evaluation process. 
Worthy of note, the expanded and formalized role of the transplant nurse coordinator has been a tremendous improvement in process. In particular, it allows the entire team to listen and comment in a manner not previously enjoyed. The nurse coordinators are well known to the team and do not wield the traditional "captain of the ship" status historically reserved for the physician. With presentation in this manner, team members that may have been intimidated can now feel free to participate fully in any discussions. The unbiased and objective presentation allows the entire team to carefully ask questions and weigh in on the decision process. The transplant nurse coordinators utilize a format (see Supplementary material) for consistency in presentation.

An additional innovation includes using technology to improve communication. Audiovisual equipment is used so that the patients' information from the form (see Supplementary material) is displayed on a large projection screen while being orally presented by the transplant nurse coordinator. This decreases the need for paper copies and increases our compliance with Health Information Portability and Accountability Act (HIPAA) regulations. Technology plays a further role as our collaborating and referral centers can readily participate in meetings and offer background information on patients that may not have been readily available in the past. Referring physicians, specialists that may have limited transplant experience, and staff caring for patients in a rehabilitation facility can now participate in real time discussions regarding the continuity of care for these complex patients. In addition, the referring centers offer comprehensive updates and follow up for the entire team about patients discharged from our care posttransplantation.

Change for our program was not limited to the multidisciplinary committee structure. Between 2004 and 2010, the medical and surgical directors who coordinated the heart failure and transplantation programs had distinct and at times conflicted approaches to patient care decisions. Frustrations among team members increased, becoming at times, transparent to patients and family members. At this juncture in time, the only forum in which the large group met as a team was at the weekly MDR. Recognizing a need for change, particularly with communication, the team members agreed that the weekly meeting alone was an inadequate platform for the dynamic decision making required for acutely ill inpatient transplant patients. Of note during this period, experts in the field and regulatory agencies alike were emphasizing the need for transplant programs to adopt organized multidisciplinary rounding as a standard of care. As a result, a plan to meet daily in the patient care conference room and to have all disciplines involved to discuss the plan of care was developed. The implementation of this plan, however, was fraught with challenges. These challenges included: adhering to a specific timeframe for rounds that ensured best representation by the multidisciplinary team members, establishing "ownership" of and a reliable mechanism for documentation of rounds, and developing the forum with emphasis on respectful collaboration. For example, coordination and communication were challenging and rounds would often occur based on a daily phone call from the surgeon to "round up the team." Clearly this led to intermittent attendance due to multiple time conflicts.

With the recruitment of new leadership in 2011 for the advanced heart failure program, the team was able to regroup and prioritize opportunities for improvement. In conjunction with changes to the weekly meeting, patient rounding at specifically designated times by the multidisciplinary team was formalized. The daily MDR represented the perfect venue through which changes in the leadership and programmatic culture could be imparted. Scott-Findlay and Golden-Biddle note that one place where the values of the setting are reflected, transmitted, and enforced is in routines such as patient rounds. ${ }^{10}$

\section{Institution of daily MDRs}

As the transition to new physician leadership occurred, many of the previously established processes were refined and new ones were created. An expectation was set that MDRs would commence daily at $2 \mathrm{pm}$ in the cardiothoracic intensive care unit (CTICU) and include a scripted dialogue in which either the nurse practitioner or physician assistant led by the intensive care unit intensivist, the attending cardiologist, and the cardiac surgeon presents the patient to the entire team. This collaborative group can also include any family members who happen to be present at the time.

MDRs are held daily. At the outset, the surgical, medical, and administrative directors showed up to round every day, earlier than the appointed time. Daily attendance was, and continues to be, taken at MDRs. Team members who do not show up, or show up late are held accountable for their lack of participation. Team members who cared deeply about their personal participation learned that they could rely on this forum for a respectful audience. Intransigent team members were gradually replaced over time. All team members and consultants have learned that they can rely on the constancy of $2 \mathrm{pm}$ MDRs to discuss plans with the entire team. After all CTICU patients have been seen, the team continues to progress through the hospital to the cardiac intensive care unit, the step down unit, and floor as necessary. With these changes, articulation of a clear plan of care has improved 
dramatically. The rounds are documented in the Electronic Medical Record (EMR) by the transplant nurse coordinator. The impact of MDR on our center and our patient outcomes was dramatic and the model developed successful to the extent that it was adopted for use for the mechanical circulatory support patient population.

A new MDR note was created in the electronic medical record (Figure 1). The transplant coordinators worked with the hospital's information technology team to create a document that would serve the needs of the patient and care team as well as meet regulatory requirements. It allows for a consistent plan of care to be documented by the team, greatly enhancing communication. It also allows the patient and/or family members an opportunity to have concerns and questions addressed formally by the team. Team members find this beneficial since historically some of these issues were not adequately identified until the post discharge phase.

Although the endeavor to change existing structure at YNHH was driven in part by provisions of regulatory policy, a newly recruited physician leadership team proved critical to its successful inception. New leadership took a fresh approach to the manner in which to implement compliance related mandates. Improvements included goals such as expanding the core members of the team to include additional ancillary services, incorporating regularly scheduled bedside rounds, adopting well thought out quality assurance and performance improvement measures, and refining existing cardiac transplant protocols to reflect best practice as defined by the International Society for Heart and Lung Transplantation. ${ }^{11}$

The 2012 Institute of Medicine discussion paper, "Core Principles and Values of Effective Team-Based Health Care"

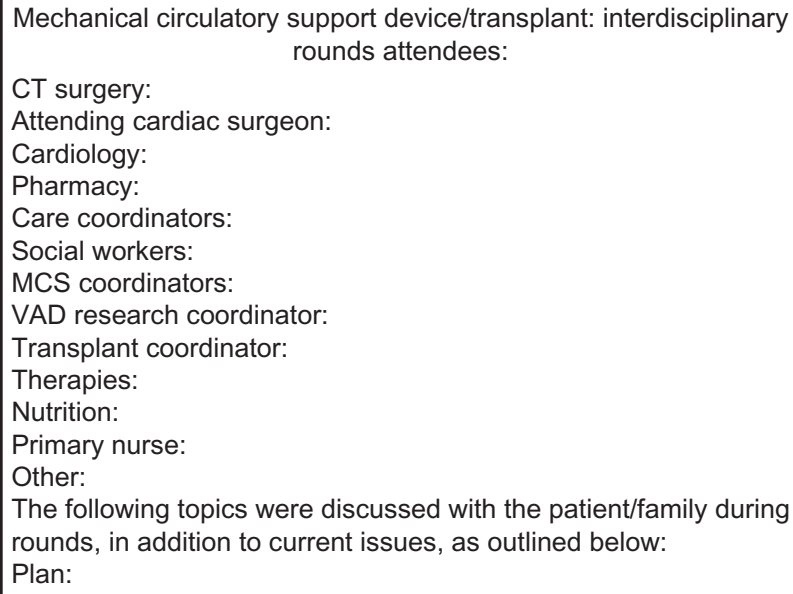

Figure I Mechanical circulatory support/transplant multidisciplinary review note. Abbreviations: CT, cardiothoracic surgeon; MCS, mechanical circulatory support; $\mathrm{VAD}$, ventricular assist device. describes the evolution in health care from "one all-knowing physician who lived in the community, made house calls and was available day and night" to the modern multifaceted health care team, as the transition from soloist to a member of an orchestra. ${ }^{12}$ In addition to this, it highlights the fact that modern health care is complex and changing at such a rapid pace that no one clinician could possibly keep current with all the new practice guidelines and research being done. Clearly, a team of experts is needed to provide safe care for complex hospitalized patients.

Even with a team of experts, however, there are challenges. Having expertise in a particular discipline does not necessarily make you an expert team member. A cardiac transplant patient being cared for in the hospital is "cared" for by many disciplines: cardiology, cardiac surgery, nursing, physician assistants, advanced practice registered nurses, pharmacists, social workers, care coordinators, physical and occupational therapists, speech and language pathologists, consultants from infectious diseases, psychiatry, and wound care specialists. Furthermore, there is an entire team "behind the scenes" including the management/leadership team from the unit in which the patient is being cared for, one or more administrative directors, performance/quality improvement managers, billing, discharge planners, utilization review, financial coordination, etc. Our MDR has multiple facets through which various members may contribute via the forum that best serves both the patient and the team while respecting individual time constraints. For example, the billing representative attends the weekly MDRC but not the bedside rounds. Revising the MDRC format and instituting formal MDR under the new leadership was a welcomed change for patients and clinicians caring for our transplant population.

\section{Quality initiatives with agreed upon metrics and transparent dashboards}

Seeking to optimize our performance, the team enlisted the assistance of the Heart and Vascular Performance Manager who capably helped with creation of a data set and establishment of metric specifications once specific quality indicators and targets were determined. The performance manager further guided the team during the bimonthly multidisciplinary Quality Assurance/Performance Improvement (QA/PI) meetings to refine the metrics and to determine the best way to display the data via the YNHH dashboard. Since the data is web based and readily available, we are able to maximize transparency and all have an opportunity to contribute as part of the QA/PI meeting format. The dashboard includes 
various heart transplant related indices, updated in real time, affording the ability to quickly identify aberrant trends and implement corrective action as needed (Figure 2). This tool enhances the ability to improve overall care to this patient population and the ability of the team to work together on common goals. The importance of a strong performance manager to support the team in setting metrics to achieve true quality improvement cannot be understated.

\section{Improved discharge planning}

Ideally, discharge planning begins upon admission. With our multifaceted MDRs, discharge planning is initiated simultaneously with initiation of care by the cardiac transplant team. Again, care is well coordinated and is an extremely important aspect of posttransplant care.

One example of the improvements realized through the organizational changes described is in our posttransplant length of stay (LOS). The median LOS from transplant to discharge in the 1-year period from July 2010 to June 2011 was 19 days, while in the 1-year period following revision of MDR, a decrease in median LOS to 12 days was noted. This change reflected a $37 \%$ decrease in the LOS from transplant to discharge (Figure 3).

Improvement was also made in the length of time from candidate evaluation to listing for transplantation. In the

\begin{tabular}{|c|c|c|c|}
\hline$\nabla$ & $<$ & Volume & Volume by month \\
\hline \multicolumn{4}{|c|}{ Volume } \\
\hline
\end{tabular}

Volume by month

Expired cases

Median LOS

Tracheostomy \%

Stroke \%

Dialysis \%

Prolonged vent \%

Unplanned return to OR \%

Pie-unplanned return to OR by months since procedure

Sternotomy \%

ABO form \%

$A B O$ verification in OR \%

Informed consent \%

Readmission by procedure date

Pie-readmission by months since procedure

Pie-complications by months since procedure

Figure 2 Program dashboard.

Abbreviations: $L O S$, length of stay; $O R$, operating room; $A B O, A B O$ blood type.
Median transplant length of stay

n1, July 2010-June 2011, 19

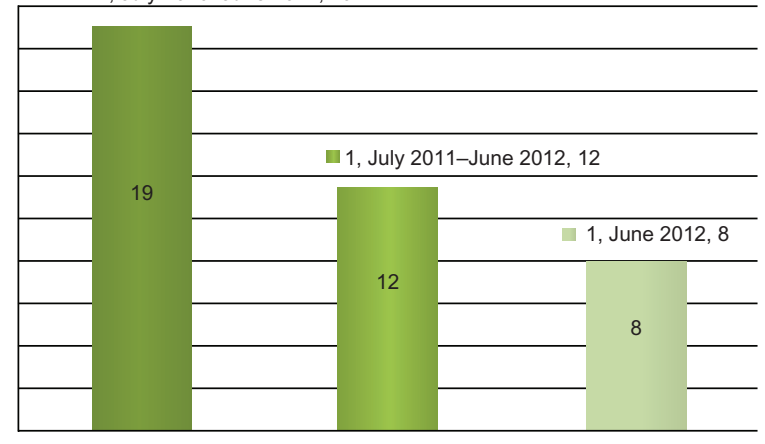

Figure 3 Median length of stay of transplant to discharge in days.

1-year period prior to changing the existing structure, the median number of days from evaluation to new listing was 78. While this number was high it clearly reflected deficiencies in the process. In the 1-year period subsequent to the change, the median number of days for completion of evaluation with arrival at a conclusive decision regarding listing was reduced to 12 . Furthermore, to date, that number has remained constant (Figure 4). Finally, a reduction by 33\% in the 30 day readmission rate for transplant patients in the 18 months following the revision to processes has become apparent (Figure 5).

\section{Future implications}

The multidisciplinary approach is not limited to the early postoperative transplant patient. Our program continues to follow patients transplanted in the early 1990s, now 20 years or more posttransplant. For these individuals, as they face the adverse effects of long-term immunosuppression, pertinent lifestyle issues and health care interventions must be addressed. Some of these individuals are known to all members of our multidisciplinary team, some not as much. We have now established a separate weekly meeting with a representative subset of the multidisciplinary team to convene for discussion of these patients. In many respects, this additional

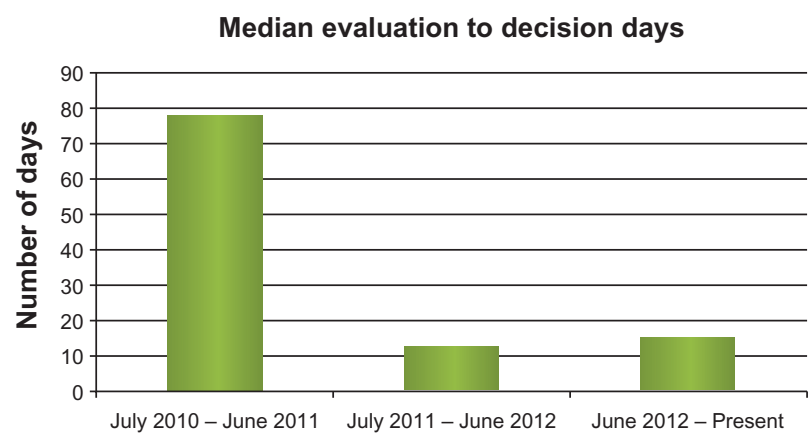

Figure 4 Median evaluation time. 


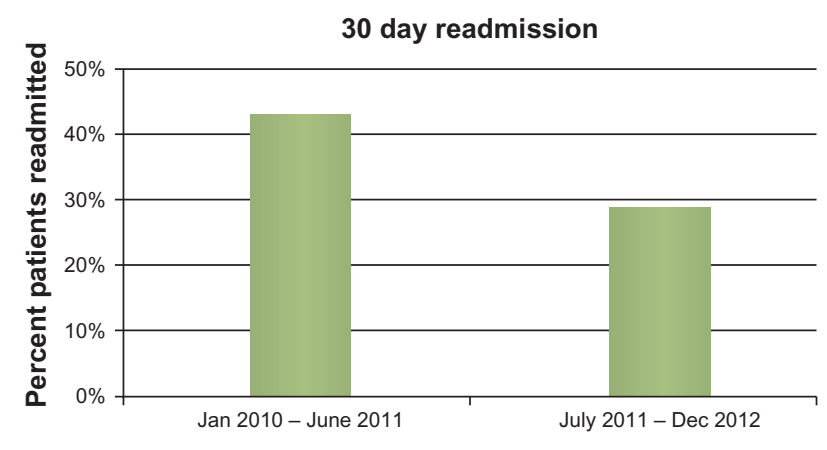

Figure $\mathbf{5} 30$ day readmission rate.

tier of team input is representative of the multidisciplinary approach come full circle. Patient related topics are not isolated to the peri-operative posttransplant period, but rather include assessment of the need and feasibility of retransplant, dialysis, palliative care, hospice, and more.

Our center is also certified by The Joint Commission as a Destination Therapy ventricular assist device (VAD) site and we use these same processes in the management of the mechanical circulatory support population. Recognizing that not all heart failure patients are transplant eligible and seeing an increasing number of patients who could benefit from mechanical circulatory support, we have applied the multidisciplinary approach as a mechanism to better serve a larger number of heart failure patients. The importance of the multidisciplinary team cannot be underscored with this extraordinarily complex population.

\section{Conclusion}

Refining the multidisciplinary approach for the management of cardiac transplant patients at our institution has had a significant impact on the quality of care delivered. The approach utilized is comprehensive, includes representation by many specialties, ancillary, and consult services and is comprised of daily MDRs as well as weekly MDRC meetings. The effects of implementation of this model are measurable. Specifically, the length of time to complete evaluations has decreased, the LOS posttransplantation has been shortened, and the 30 day all cause hospital readmission rate has declined. Additionally, this model has implications for the long-term survival of cardiac transplant patients as well as those patients under consideration for mechanical circulatory support.

\section{Acknowledgment}

The authors wish to acknowledge the extraordinary care provided by the entire team at Yale-New Haven Hospital and the administrative leaders who support our program.

\section{Disclosure}

The authors report no conflict of interest in this work.

\section{References}

1. IHI.org. IHI Improvement map multidisciplinary rounding [webpage on the Internet]. Available from: http://app.ihi.org/imap/ tool/\#Process=fe8c7382-b23a-4ef2-a49e-5e8e779875b4. Accessed July 1, 2013.

2. National Quality Forum. Effective communication and care coordination [webpage on the Internet]. Available from: http://www.qualityforum. org/Topics/Effective_Communication_and_Care_Coordination.aspx. Washington, DC: National Quality Forum. Accessed July 1, 2013.

3. Institute for Healthcare Improvement [homepage on the Internet]. Available from: http://app.ihi.org/imap/tool/\#. Accessed July 1, 2013.

4. Triggle N. Call to raise nurses' profile on ward rounds. Nurs Manag (Harrow). 2012;19(7):6-7.

5. Wensing M, Wollersheim H, Grol R. Organizational interventions to implement improvements in patient care: a structured review of reviews. Implement Sci. 2006;1:2.

6. Kim MM, Barnato AE, Angus DC, Fleisher LF, Kahn JM. The effect of multidisciplinary care teams on intensive care unit mortality. Arch Intern Med. 2010;170(4):369-376.

7. Sen A, Xiao Y, Lee S-A, et al. Daily multidisciplinary discharge rounds in a trauma center: a little time, well spent. $J$ Trauma. 2009;66(3): $880-887$.

8. Committee on Quality of Health Care in America. Crossing the Quality Chasm: A New Health System for the 21st Century. Institute of Medicine; Washington, DC: National Academy Press. Available from: http://www.nap.edu/books/0309072808/html/. Accessed July 1, 2013.

9. Gray JE, Davis DA, Pursley DM, et al. Network analysis of team structure in the neonatal intensive care unit. Pediatrics. 2010;125(6): e1460-e1467.

10. Scott-Findlay S, Golden-Biddle K. Understanding how organizational culture shapes research use. J Nurs Adm. 2005;35(7-8):359-365.

11. Costanzo M, et al. The ISHLT Guidelines For The Care Of Heart Transplant Recipients. The Journal of Heart and Lung Transplantation. 2010;29(8):914-956. Available from: http://ishlt.org/ContentDocuments/JHLTAugust10ISHLT_TransplGuidelinesHT.pdf. Accessed on July 22, 2013.

12. Mitchell P, Wynia M, Golden R, et al. 2012. Core Principles and Values of Effective Team-Based Health Care. Washington, DC: Institute of Medicine; 2012. Available from: http://www.iom.edu/tbc. Accessed July $1,2013$. 


\section{Supplementary material}

Yale Center for Advanced Heart Failure, Mechanical Circulatory Support and Heart Transplantation

Transplant Evaluation Form

Patient Name:

MRN:

Address:

Birth Date:

Age:

Hgt: Weight: BMI:

Home Phone: Cell:

Primary MD: $\quad$ Primary Phone:

Referring MD: $\quad$ Referring MD Phone:

Primary Insurance: Secondary Insurance:

SS\#:

Blood Group: $\quad$ RH Group:

Referral Diagnosis:

Secondary Diagnosis:

HPI:

PMH:

Social Hx:

Surgical History:

Allergies:

Medications:

Echocardiogram:

\begin{tabular}{|l|l|l|}
\hline Date of Lab: & RVEF & LVEF \\
\hline
\end{tabular}

Impression:

Right Heart Catheterization

\begin{tabular}{|l|l|l|l|l|l|l|}
\hline Date of Lab & RA & PA & PAm & PCWP & CO/CI & PVR \\
\hline
\end{tabular}

Left Heart Catheterization

\begin{tabular}{|l|l|l|l|l|}
\hline Date of Lab & RCA & LAD & LCX & LCM \\
\hline
\end{tabular}

PRA - Serology

\begin{tabular}{|l|l|l|l|l|l|}
\hline Date of lab & Pra_1_CDC & Pra_2_CDC & Pra_1_FLOW & Pra_2_FLOW & cPRA \\
\hline
\end{tabular}

PFT: Date of Lab:

\begin{tabular}{|l|l|l|l|l|l|l|l|l|l|}
\hline FVC/FVC $\%$ & FEV1 & FEV $1 \%$ & RV & RV $\%$ & TLC & TLC $\%$ & FRC $\%$ PL & DLCO $\%$ & DLCO/VA $\%$ \\
\hline
\end{tabular}


CPETT Date of Study: Not performed

\begin{tabular}{|l|l|l|l|l|l|l|l|}
\hline $\begin{array}{l}\mathrm{VO}^{2} \mathrm{Max} \\
(\mathrm{ml} / \mathrm{kg} / \mathrm{min})\end{array}$ & $\begin{array}{l}\mathrm{VO}^{2} \mathrm{Max} \\
(\mathrm{ml} / \mathrm{kg} / \mathrm{min}) \mathrm{AT}\end{array}$ & RER AT & RER Max & HRMax $\%$ & BP Base & BP Max & $\begin{array}{l}\text { Exercise } \\
\text { Time }\end{array}$ \\
\hline
\end{tabular}

Surgery Consult:

Social Work Consult:

Psych Consult:

Transplant Evaluation Form

Patient Name:

MRN:

CXR:

Abdominal U/S:

Carotid U/S:

ABI:

Chest CT:

Colonoscopy

Dental Clearance:

Nutrition Consult:

Pharmacology Consult:

Laboratory Data:

\begin{tabular}{|l|l|l|l|l|l|l|l|l|l|}
\hline WBC & HgB & HCT & PLAT & PT/PTT & INR & GLU & BUN & CR & NA \\
\hline
\end{tabular}

\begin{tabular}{|l|l|l|l|l|l|l|l|l|l|}
\hline $\mathrm{K}$ & $\mathrm{CL}$ & $\mathrm{CO}^{2}$ & $\mathrm{CA}$ & PO4 & MG & U ACID & CPK & T.PRO & ALB \\
\hline
\end{tabular}

\begin{tabular}{|l|l|l|l|l|l|l|l|l|l|}
\hline T.BILI & D.BILI & ALT & AST & ALK P & AMY & LIPASE & EFT & T4 & TSH \\
\hline
\end{tabular}

\begin{tabular}{|l|l|l|l|l|l|l|l|l|l|}
\hline CHOL & HDL & LDL & TRIG & FERRITIN & PSA & CRCL & GFR & RF & VDRL \\
\hline
\end{tabular}

\begin{tabular}{|l|l|l|l|l|l|l|l|}
\hline ANA & HBsAg & HBsAb & HBcAb & HepC Ab & HepA Ab & HIV & Toxo \\
\hline
\end{tabular}

\begin{tabular}{|l|l|l|l|l|}
\hline CMV IgG & CMV IgM & EBV & H SIMPLEX & HgbA1C \\
\hline
\end{tabular}




\section{QUANT \\ IG}

SPEP

$\mathrm{U} / \mathrm{A}$

PPD

\begin{tabular}{|l|l|}
\hline Hep A Vaccination & Hep B Vaccination \\
$\# 1$ & $\# 1$ \\
\hline
\end{tabular}

Journal of Multidisciplinary Healthcare

Dovepress

\section{Publish your work in this journal}

The Journal of Multidisciplinary Healthcare is an international, peerreviewed open-access journal that aims to represent and publish research in healthcare areas delivered by practitioners of different disciplines. This includes studies and reviews conducted by multidisciplinary teams as well as research which evaluates the results or conduct of such teams or and welcomes submission from practitioners at all levels, from all over the world. The manuscript management system is completely online and includes a very quick and fair peer-review system. Visit http://www.dovepress.com/testimonials.php to read real quotes from published authors.

Submit your manuscript here: http://www.dovepress.com/journal-of-multidisciplinary-healthcare-journal 